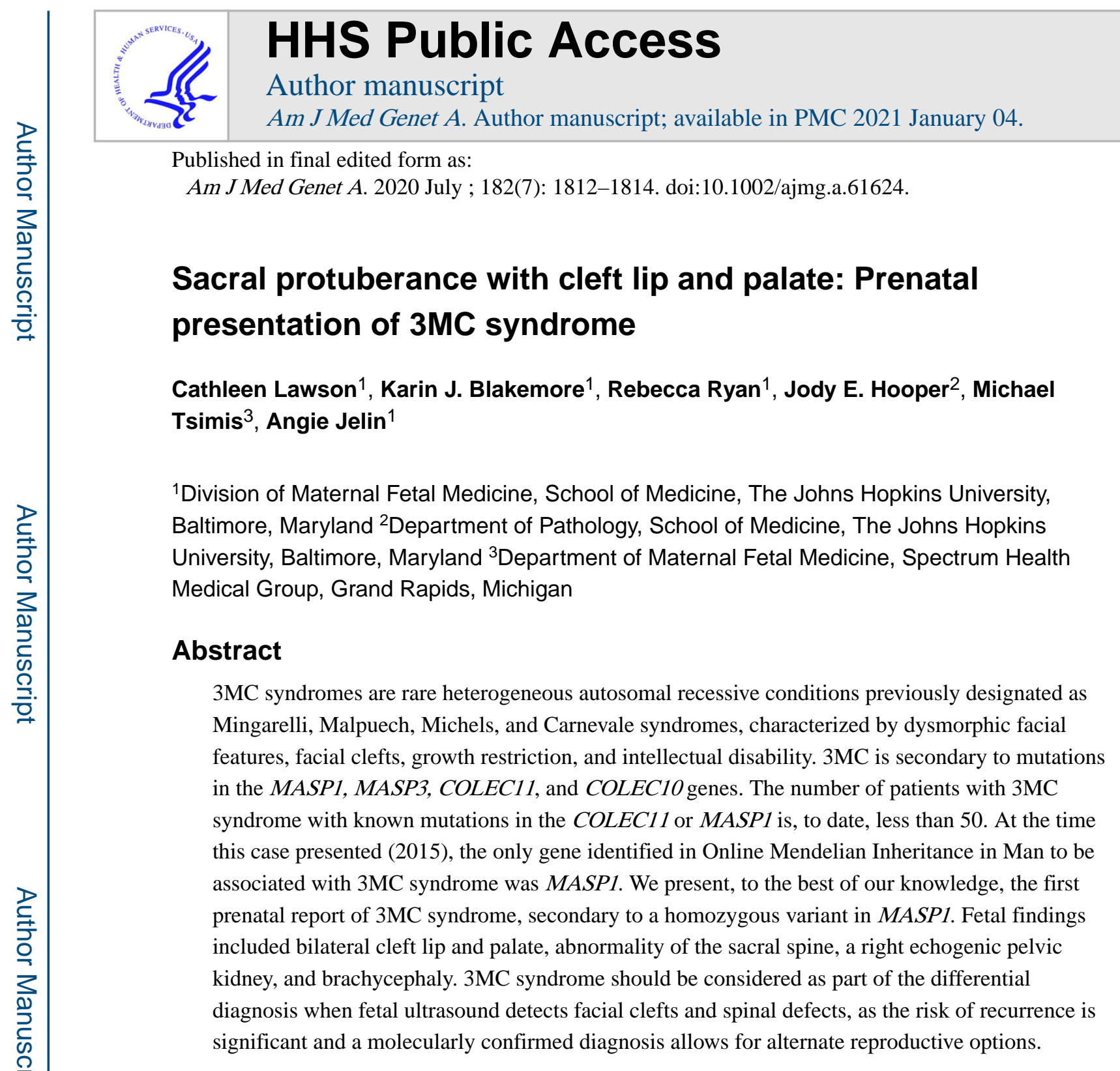

\title{
Keywords
}

3MC syndrome; cleft lip; cleft palate; fetal; $M A S P 1$; sacral protuberance

\footnotetext{
Correspondence: Cathleen Lawson, Division of Maternal Fetal Medicine, School of Medicine, Johns Hopkins Hospital, 600 N. Wolfe St. Nelson 255, Baltimore, MD 21287. clawso15@jhmi.edu.

AUTHOR CONTRIBUTIONS

Cathleen Lawson provided genetic counseling to the patient, developed the differential diagnosis, coordinated genetic testing, and drafted the case report for publication.

Karin J. Blakemore reviewed the fetal images and performed a dysmorphology examination of the fetus at delivery and revised the work for intellectual content for publication.

Rebecca Ryan performed the obstetric sonogram. Jody E. Hooper performed the fetal autopsy. Michael Tsimis reviewed the fetal ultrasound images and counseled the patient.

Angie Jelin substantially contributed to the interpretation of the phenotype, drafted and revised the work for intellectual content, and approved the final version.

CONFLICT OF INTEREST

The authors declare no conflicts of interest.

DATA AVAILABILITY STATEMENT

Data sharing is not applicable to this article as no new data were created or analyzed in this study.
} 


\section{1| CASE REPORT}

A 38-year-old, gravida 5, para 0040, Asian Indian woman was referred to our center at 25 weeks gestation for multiple fetal anomalies found by ultrasonography and MRI at an outside center. Her husband is a 40-year-old Asian Indian male. The couple is nonconsanguineous. The patient's four prior pregnancies all resulted in first trimester miscarriage and the current pregnancy was conceived by in vitro fertilization (IVF) with their own sperm and ovum. Prior to her visit in our center a first trimester ultrasound performed at 12 weeks gestation revealed a nuchal translucency of $1.6 \mathrm{~mm}$ and present nasal bone. Cell-free DNA screening for chromosomes 21, 18, 13, X, and five microdeletion syndromes (22q11.2 deletion syndrome, 1p36 deletion syndrome, Angelman syndrome, Prader-Willi-syndrome, and Cri-du-chat syndrome) was negative. An obstetrical ultrasound at 19 weeks 4 days was reported as normal, although the fetal anatomic survey was incomplete due to lack of visualization. An obstetrical ultrasound and fetal echocardiogram performed at 21 weeks 6 days reported cleft lip and palate, absent right kidney, and a sacral protuberance. Amniocentesis was performed and revealed a 46,XX karyotype, AF-AFP of $0.82 \mathrm{MoM}$, and normal chromosomal microarray. Fetal MRI and ultrasound at 23 weeks revealed bilateral cleft lip and palate, a sacral neural tube defect (L5-S1) with nerve roots extending to the defect and irregular spinal canal accompanied by small irregular soft tissue protuberance, distended gallbladder with internal septations, small echogenic right kidney, and unusual contour to the calvarium suggestive of possible early craniosynostosis.

At 25 weeks gestation an ultrasound evaluation in our center affirmed bilateral cleft lip and palate (Figure 1), abnormality of the sacral spine (Figure 2), a right echogenic pelvic kidney and brachycephaly. Specifically, there was an echolucent area within the spinal canal at the level of L4-S1 and a dorsal cutaneous/subcutaneous soft tissue protrusion just above the coccyx. Spinal dysraphism also appeared to be present from $\sim$ S1-S4. The overlying skin surface appeared intact. Fetal growth was consistent with dating by IVF conception. The patient proceeded with pregnancy termination immediately following this ultrasound. She consented for fetal autopsy, which noted a mid-line cleft lip and palate, mild hypertelorism, abnormally widened metopic sutures, widened anterior fontanelle, posterior rotation of the left ear, and an overriding fifth toe of the right foot. The only internal organ abnormality was a bilobed right lung. The fetal kidneys were unremarkable. The presence of a closed spinal dysraphism was confirmed, with nerve roots and vessels in the low lumbosacral levels contained within loose connective tissue and dense fibrous tissue in direct continuity with subcutaneous soft tissue and an unremarkable skin surface covering the sacrococcygeal protuberance (Figure 2). Additional features of 3MC syndrome, including blepharophimosis, blepharoptosis, and radioulnar synostosis, were not identified and could be difficult to identify in a fetus.

The following search terms were entered into Online Mendelian Inheritance in Man: cleft lip, hypertelorism, anterior fontanelle, and sacral spina bifida. Review of the results suggested the most likely diagnosis to be $3 \mathrm{MC}$ syndrome. The results of this query resulted in the following differential diagnosis: $3 \mathrm{MC}$ syndrome, acrofrontofacionasal dysostosis 2 , autosomal recessive Robinow syndrome, Opitz-Kaveggia syndrome, Feingold syndrome 1, van Maldergem syndrome 1, and van Maldergem syndrome 2. Given the small amount of 
fetal DNA remaining from the amniocentesis and the likelihood for 3MC syndrome, direct sequencing using next generation short base pair read sequencing of the MASP1 gene was performed on parental blood, and both members of the couple were found to be heterozygous for a nonsense mutation in $M A S P 1$ (c.1779G>A). Polymerase chain reaction analysis for analysis of exon 11 of $M A S P 1$ of the remaining fetal DNA confirmed the fetus to be homozygous for the c.1779G>A, p.W593X (NM_139125.3) mutation in the MASP1 gene, confirming the diagnosis of $3 \mathrm{MC}$ syndrome. This gene codes for the protein mannoseassociated serine protease 1. This nonsense mutation causes loss of function and is a rare variant with phenotype specific to this disease. The laboratory reported this variant as pathogenic, consistent with American College of Medical Genetics and Genomics variant classification (Richards et al., 2008). Based on our case, the laboratory has also reported this variant as pathogenic in ClinVar. The population frequency of this variant in ExAC is $1.0 \times \mathrm{e}$ -5 and the dbSNP code is rs 549330397.

\section{2 | CONCLUSION}

This case illustrates a collection of prenatal findings detectable by ultrasonography, which together, should suggest the diagnosis of $3 \mathrm{MC}$ syndrome and allow for targeted molecular genetic analysis. Most notably, the unusual finding of a caudal appendage of the spine in combination with the fetal cleft lip/palate should prompt evaluation for further evidence of $3 \mathrm{MC}$ syndrome. Prenatally, these additional dysmorphic features would similarly prompt an evaluation for this syndrome.

The characteristic features of 3MC are facial dysmorphism which includes hypertelorism, blepharophimosis, blepharoptosis, and high arched eyebrows (70-95\% of cases); cleft lip/ palate (50\% of cases); postnatal growth restriction (60\% of cases), hearing loss (65\% of cases); intellectual disability of variable severity (60\% of cases). Less common features are caudal appendage (37\% of cases); craniosynostosis, radioulnar synostosis, and genital and vesicorenal anomalies (20-30\% of cases); and anterior chamber anomalies and cardiac defects ( $<20 \%$ of cases) (Rooryck et al., 2011). As previously stated, it is secondary to mutations in the MASP1, MASP3, COLEC11, and COLEC10 genes (Munye et al., 2017; Urquhart et al., 2016). Although difficult to determine the phenotypic spectrum of COLEC11 and MASP1 mutations because of the limited number of patients, it has been reported (Atik et al., 2015) that cleft lip/palate, hearing loss, vesicorenal anomalies, genital anomalies, and caudal appendages were more frequent in patients with variants in MASP1.

The diagnosis of 3MC syndrome in our case allowed for accurate recurrence risk counseling and expanded the available reproductive options to reduce the risk of recurrence. The pregnancy had been conceived by IVF and the couple reported having cryopreserved embryos. With a molecularly confirmed diagnosis, preimplantation genetic testing for the couple's cryopreserved embryos is now an option the couple may pursue.

\section{REFERENCES}

Atik T, Koparir A, Bademci G, Foster J II, Altunoglu U, Mutlu GY, ... Tekin M (2015). Novel MASP1 mutations are associated with an expanded phenotype in 3MC1 syndrome. Orphanet Journal of Rare Diseases, 10, 128. [PubMed: 26419238] 
Munye M, Diaz-font A, Ocaka L, Henriksen M, Lees M, Brady A, ... Hernandez-Hernandez V (2017). COLEC1O is mutated in $3 \mathrm{MC}$ patients and regulates early craniofacial development. PLoS Genetics, 13(3), e1006679. [PubMed: 28301481]

Richards CS, Bale S, Bellissimo DB, Das S, Grody WW, Hegde MR, ... Molecular Subcommittee of the ACMG Laboratory Quality Assurance Committee. (2008). ACMG recommendations for standards for interpretation and reporting of sequence variations: Revisions 2007. Genetics in Medicine, 10, 294-300. [PubMed: 18414213]

Rooryck C, Diaz-Font A, Osborn D, Chabchoub E, Hernandez-Hernandez V, Shamseldin H, ... Beales $\mathrm{P}$ (2011). Mutations in lectin complement pathway genes COLEC11 and MASP1 cause 3MC syndrome. Nature Genetics, 43(3), 197-203. [PubMed: 21258343]

Urquhart J, Roberts R, de Silva D, Shalev S, Chervinsky E, Nampoothiri S, ... Clayton-Smith J (2016). Exploring the genetic basis of 3MC syndrome: Findings in 12 further families. American Journal of Medical Genetics-Part A, 170A, 1216-1224. [PubMed: 26789649] 


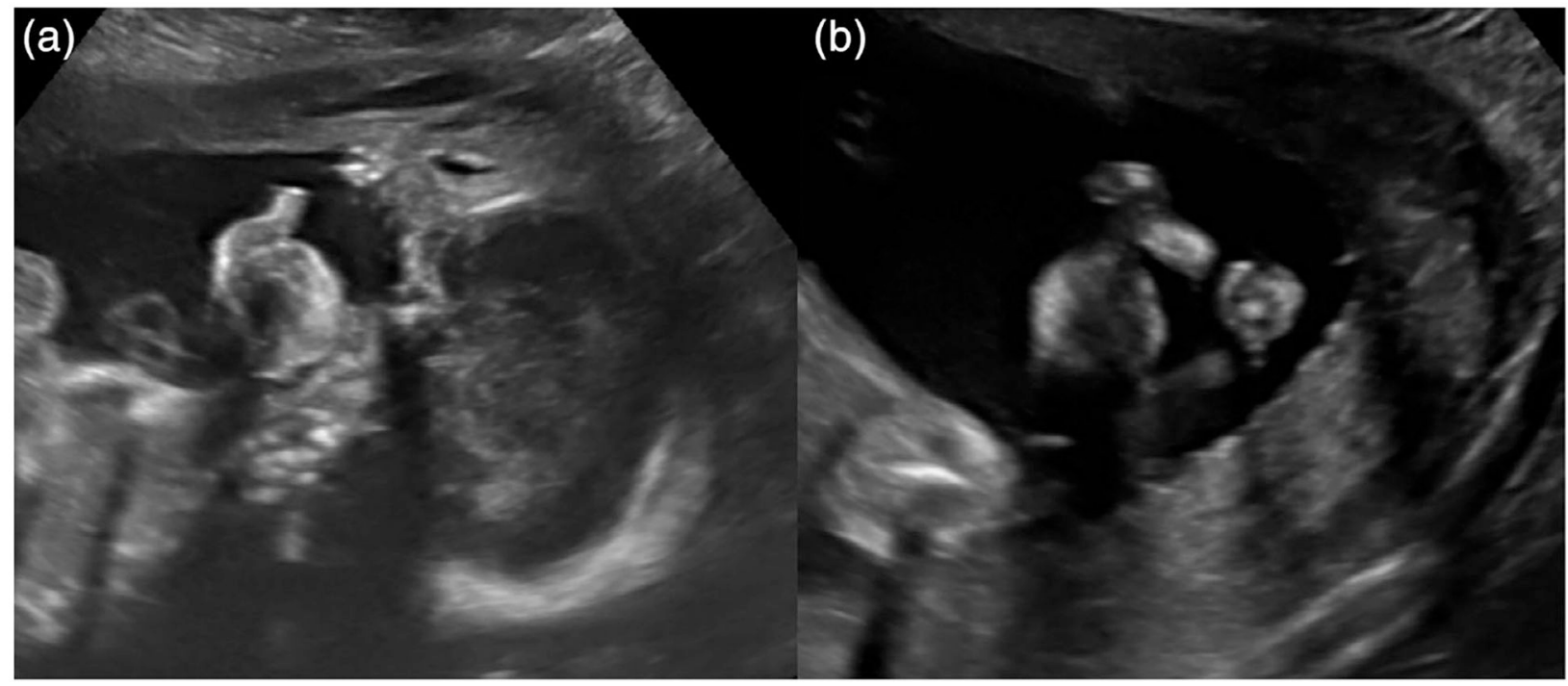

FIGURE 1.

Coronal bilateral cleft lip at 25 weeks gestation 

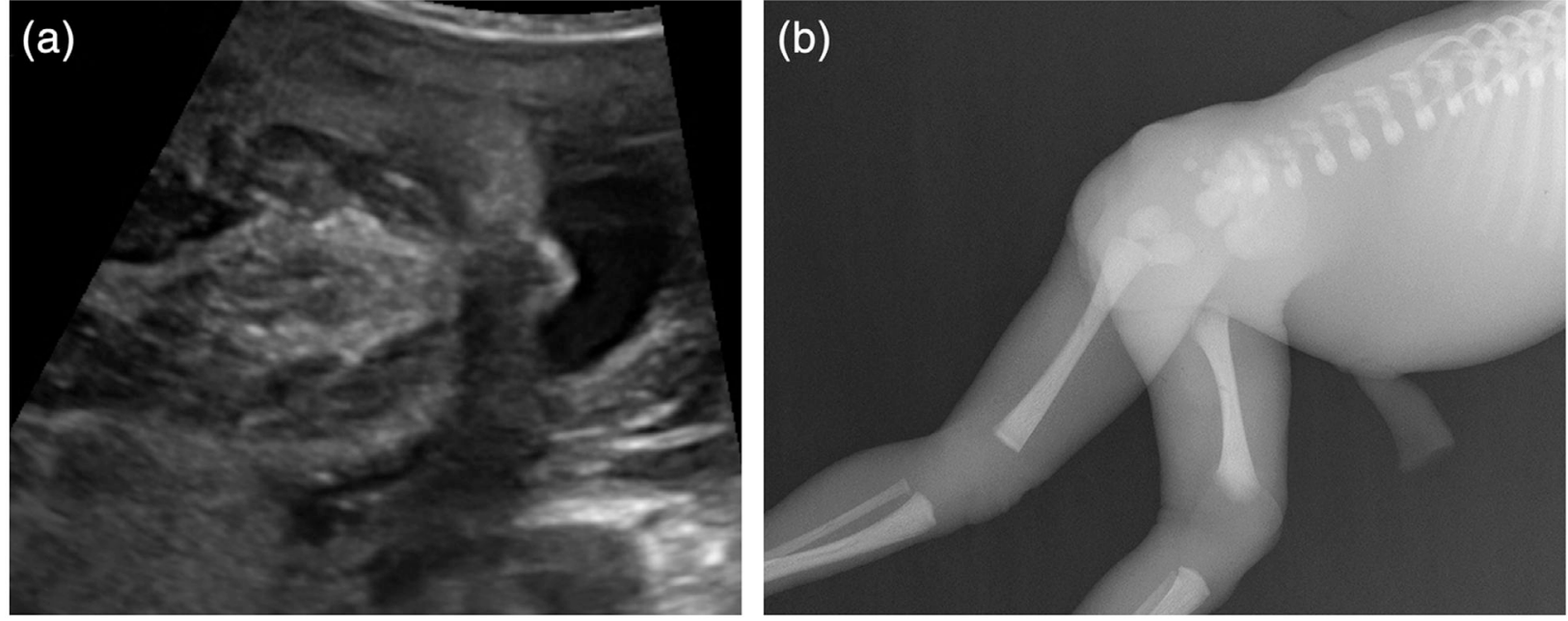

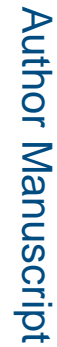

FIGURE 2.

Sacral protuberance at 25 weeks gestation 